\title{
Joint Numerical Range of Matrix Polynomials
}

\author{
Ahmed M. Sabir \\ College of Sciences \\ University of Salahaddin \\ Received on: 26/11/2007 \\ Accepted on: 11/06/2008
}

\begin{abstract}
Some algebraic properties of the sharp points of the joint numerical range of a matrix polynomials are the main subject of this paper. We also consider isolated points of the joint numerical range of matrix polynomials.

Key words: joint numerical range, matrix polynomial, sharp points.

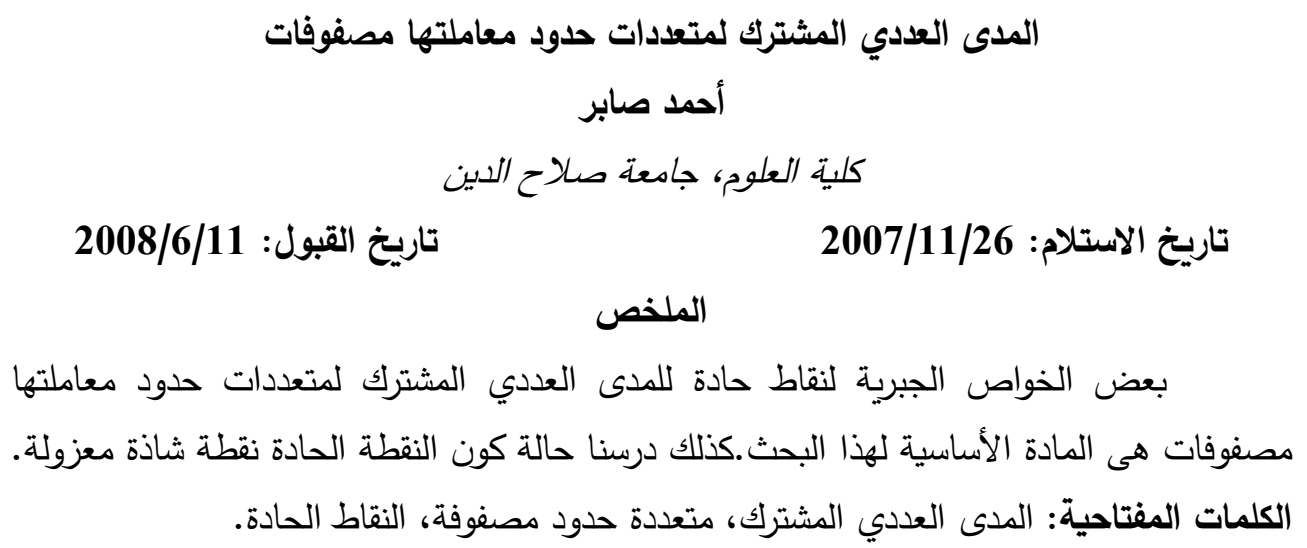

\section{1- Introduction:}

Let $A \in M_{n}$ be the algebra of $n \times n$ complex matrices. The classical numerical range of $A$ is the set of a complex numbers $W(A)=\left\{x^{*} A x\right.$ : $\mathrm{x} \in \mathrm{C}^{\mathrm{n}}, \mathrm{x}^{*} \mathrm{x}=1$ \} where $\mathrm{C}^{\mathrm{n}}$ vector space (over $\mathrm{C}$ ) of complex n-vectors [6]. There has been many generalizations and applications of the classical numerical range, see, for example[6]. In the following, we consider a generalization of the classical numerical range. Suppose $\quad p(\lambda)=A_{m} \lambda^{m}+A_{m-1} \lambda^{m-1}+\ldots+A_{1} \lambda+A_{0}$ is a matrix polynomial, where $\mathrm{A}_{0}, \mathrm{~A}_{1}, \mathrm{~A}_{2}, \ldots, \mathrm{A}_{\mathrm{m}} \in \mathrm{M}_{\mathrm{n}}$ and $\lambda$ is a complex variable. Define the joint numerical range of $p(\lambda)$ as $J N R(p(\lambda))=\left\{\left(x^{*} A_{0} x, x^{*} A_{1} x, \ldots, x^{*} A_{m} x\right): x \in C^{n}: x^{*} x=1\right\}$ [9].

This generalized joint numerical range has been discussed by [9]. On the other hand The joint numerical range of matrix polynomials, being a continuous image of the unit sphere, is compact and connected but not necessarily convex; see Binding and $\mathrm{Li}$ [3]. Its convex hull is denoted by co $\{J N R(p(\lambda))\}$ and it plays an important role in the study of damped vibration 
systems, with a finite number of degree of freedom [7] and it is useful in various theoretical and applied subjects (see[1,2,3,4 and 5]) and their references. The aim of this paper is to give some algebraic properties of the sharp points of the joint numerical range of matrix polynomials, we also consider an isolated point of the joint numerical range of $p(\lambda)$.The rest of this paper is organized as follows: In section 2, we present definitions and some basic results which will be used in this paper. In section 3, we prove that if $\lambda_{0}$ is a sharp point of the joint numerical range of the linear pencil $A_{1} \lambda_{1}-B_{1}, A_{2} \lambda_{2}-B_{2}, \ldots, A_{m} \lambda_{m}-B_{m}$ then zero is a sharp point of $J N R\left(A_{1} \lambda_{o}-B_{1}, A_{2} \lambda_{o}-B_{2}, \ldots, A_{m} \lambda_{o}-B_{m}\right)$.and if $\lambda_{0}$ is an isolated point of $J N R(p(\lambda))$, then $p\left(\lambda_{0}\right)=0$.

\section{2- Preliminaries:}

In this section, we present some definitions and basic results on joint numerical ranges of matrix polynomials.

\section{Definition 2.1 [8]}

A point $\lambda_{0} \in N R(p(\lambda))$ is called a sharp point of $N R(p(\lambda))$ if for a connected component $w_{s}$ of $N R(p(\lambda))$ there exists a disk centered at $\lambda_{0}$ and with radius $\mathrm{r} S\left(\lambda_{0}, r\right), \mathrm{r}>0$ and angles $\phi_{1}$ and $\phi_{2}$ with $0 \leq \phi_{1}<\phi_{2} \leq 2 \pi$ such that

$$
\operatorname{Re}\left(e^{i \theta} \lambda_{0}\right)=\max \left\{\operatorname{Re} z: e^{-i \theta} z \in w_{s}(p(\lambda)) \cap S\left(\lambda_{0}, r\right)\right\},
$$

for all $\theta \in\left[\phi_{1}, \phi_{2}\right]$.

\section{Definition 2.2 [6]}

A matrix $A \in M_{n}$ is said to be unitary if $A^{*} A=I$, if in addition $A \in M_{n}(R)$ then A is said to be real orthogonal.

\section{Theorem 2.3[6]}

If $A \in M_{n}$ the following are equivalent.

a) $\mathrm{A}$ is unitary.

b) $\mathrm{A}$ is non singular and $A^{*}=A^{-1}$.

c) $A A^{*}=I$.

d) $A^{*}$ Is unitary.

\section{Proposition 2.4 [6]}

Suppose that $Q(\lambda, t)=C_{m(t)} \lambda^{m}+C_{m-1(t)} \lambda^{m-1}+\ldots+C_{1(t)} \lambda+C_{0} \quad$ is $\quad$ a polynomial matrix in $\lambda$ where the coefficient $C_{j(t)}$ depend continuously on the parameter $0 \leq t<\varepsilon$ and $C_{m(t)} \neq 0$ for every $o \leq t<\varepsilon$ then $\mathrm{m}$ roots $\lambda_{\mathrm{j}(\mathrm{t})}$; $j=1,2, \ldots, m$ of the equation $Q(\lambda, t)=0$ are continuous functions in $t \in[0, \varepsilon)$. 


\section{Definition 2.5 [6]}

An n-by-n Hermitiat matrix $A$ is said to be positive definite if $x^{*} A x>0$ for all non-zero $x \in C^{n}$.

\section{Definition 2.6 [6]}

A matrix $B \in M_{n}$ is said to be positive semi-definite if $x^{*} B x \geq 0$ for all $\mathrm{x} \in \mathrm{C}^{\mathrm{n}}$

\section{Definition 2.7 [6]}

The matrix adjoint $A^{*}$ of $A \in M_{n}(C)$ is define by $A^{*}=A^{-T}$ where $A^{-}$is the component-wise conjugate, and $\mathrm{A}^{\mathrm{T}}$ is the transpose of $\mathrm{A}$.

\section{Definition 2.8 [6]}

The matrix $A \in M_{n}(C)$ is said to be Hermition if $A=A^{*}$, it is skewHermition if $A=-A^{*}$ and for any $A \in M_{n}(C)$ can be written $A=\left(A+A^{*}\right) / 2$ $+\left(\mathrm{A}-\mathrm{A}^{*}\right) / 2=\mathrm{H}(\mathrm{A})+\mathrm{S}(\mathrm{A})$ where $\mathrm{H}(\mathrm{A})=\left(\mathrm{A}+\mathrm{A}^{*}\right) / 2$ the Hermition part of $\mathrm{A}$, and $\mathrm{S}(\mathrm{A})=\left(\mathrm{A}-\mathrm{A}^{*}\right) / 2$ is the skew-Hermition part of $\mathrm{A}$.

\section{Definition 2.9 [6]}

Let $p_{0}$ be an element of anon-empty set $\mathrm{A}$, we say that $p_{0}$ is an isolated point of A if $\exists N_{r}\left(p_{0}\right)$ such that $N_{r}\left(p_{0}\right) \cap A=\left\{p_{0}\right\}$

\section{3- Properties of Sharp points}

In the following, we will restrict ourselves to the definition of sharp points. The next theorem gives a connection of these points with respect to the origin as a joint numerical range of matrix polynomials.

\section{Theorem 3.1}

Suppose that $x_{o}$ is a unit vector such that $0=x_{o}^{*} A_{1} x, \ldots, 0=x_{o}^{*} A_{n} x$ belongs to the joint numerical range of $A_{1}, A_{2}, \ldots A_{n} \in M_{n}$ if $x^{*} A_{1} x, \ldots, x^{*} A_{n} x$ have non-negative real parts for all $\mathrm{x}$ of the neighborhood $S\left(x_{o}, \varepsilon\right)=\left\{x \in c^{n}:\left\|x-x_{o}\right\|_{2}<\varepsilon\right\}$ then $A_{1}+A_{1}^{*}, \ldots, A_{n}+A_{n}^{*}$ are positive semidefinite.

\section{Proof:}

Let $\lambda_{1}, \lambda_{2}, \ldots, \lambda_{n}$ be the eigenvalues of each of the Hermitian matrices $A_{1}+A_{1}^{*}, \ldots, A_{n}+A_{n}^{*}$. We see that $x^{*}\left(A_{1}+A_{1}^{*}\right) x=y_{1}^{*} D y_{1}, \ldots, x^{*}\left(A_{n}+A_{n}^{*}\right) x=y_{n}^{*} D y_{n}$ where $D=\operatorname{diag}\left(\lambda_{1}, \lambda_{2}, \ldots, \lambda_{n}\right), y=u^{*} x$ and $\mathrm{u}$ is a unitary matrix. For $y_{o}=u^{*} x_{o}=\left[y_{1}, y_{2}, \ldots, y_{n}\right]^{T} \quad$ we have $y_{o}^{*} D y_{o}=\lambda_{1}\left|y_{1}\right|^{2}+\ldots+\lambda_{n}\left|y_{n}\right|^{2}=0 \quad \ldots(*)$, since, $\left\|y-y_{o}\right\|_{2}=\left\|u^{*}\right\|_{2}\left\|x-x_{o}\right\|_{2}=\left\|x-x_{o}\right\|_{2}$, and $\operatorname{Re}\left(x^{*} A_{1} x\right) \geq 0, \ldots, \operatorname{Re}\left(x^{*} A_{n} x\right) \geq 0$ for all $\mathrm{x} \in \mathrm{S}\left(\mathrm{x}_{\mathrm{o}}, \varepsilon\right)$, there exists a neighborhood $\mathrm{S}\left(\mathrm{y}_{0}, \varepsilon\right)$ such that $y^{*} D y \geq 0$ for any $y \in S\left(y_{o}, \varepsilon\right)$. Now in (*) assume that $\lambda_{k}<0$ consider the 
vector $y_{\delta}=y_{o}+\delta e_{k}=\left[y_{1} \ldots y_{k}+\delta \ldots y_{n}\right]^{T}$ where $\delta \in \mathrm{C}$ such that $0<|\delta|<\varepsilon$ and $\left|y_{k}+\delta\right|>\left|y_{k}\right|$.Then for a vector $y_{\delta}$ of the neighborhood $S\left(y_{o}, \varepsilon\right)$ we have $y_{\delta}^{*} D y_{\delta}=\lambda_{1}\left|y_{1}\right|^{2}+\ldots+\lambda_{k}\left|y_{k}+\delta\right|^{2}+\ldots+\lambda_{n}\left|y_{n}\right|^{2}=\lambda_{k}\left(\left|y_{k}+\delta\right|^{2}+\left|y_{k}\right|^{2}\right)<0$

which is contradiction. Therefore $\lambda_{k} \geq 0$ for $\mathrm{k}=1,2, \ldots \mathrm{n}$ and $D \geq 0$. Hence the matrices $A_{1}+A_{1}^{*}, \ldots, A_{n}+A_{n}^{*}$ are positive semi-definite.

\section{Theorem 3.2}

Suppose $x_{o}$ is a unit vector such that $0=x_{o}^{*} A_{1} x, \ldots, 0=x_{o}^{*} A_{n} x$ belongs to the joint numerical range of $A_{1}, A_{2}, \ldots A_{n} \in M_{n}$.If $x^{*} A_{1} x=0, \ldots, x^{*} A_{n} x=0$ for all $x \in S\left(x_{o}, \varepsilon\right)$ then $A_{1}=A_{2}=\ldots=A_{n}=0$.

\section{Proof:}

For the matrices $A_{1}, A_{2}, \ldots, A_{n}$ we consider the Hermitian matrices, $\mathrm{H}\left(\mathrm{A}_{1}\right)=\frac{1}{2}\left(\mathrm{~A}_{1}+\mathrm{A}_{2} *\right), \mathrm{H}\left(\mathrm{A}_{2}\right)=\frac{1}{2}\left(\mathrm{~A}_{2}+\mathrm{A}_{2} *\right), \ldots, \mathrm{H}\left(\mathrm{A}_{\mathrm{n}}\right)=\frac{1}{2}\left(\mathrm{~A}_{\mathrm{n}}+\mathrm{A}_{\mathrm{n}} *\right)$ and $\mathrm{S}\left(\mathrm{A}_{1}\right)=\frac{1}{2}\left(\mathrm{~A}_{1} *-\mathrm{A}_{1}\right), \mathrm{S}\left(\mathrm{A}_{2}\right)=\frac{1}{2}\left(\mathrm{~A}_{2} *-\mathrm{A}_{2}\right), \ldots, \mathrm{S}\left(\mathrm{A}_{\mathrm{n}}\right)=\frac{1}{2}\left(\mathrm{~A}_{\mathrm{n}} *-\mathrm{A}_{\mathrm{n}}\right)$ then from the hypotheses that $x_{o}^{*} A_{1} x_{o}=0, \ldots, x_{o}^{*} A_{n} x_{o}=0$ and $x^{*} A_{1} x=0, \ldots, x^{*} A_{n} x=0$ for any $x \in S\left(x_{o}, \varepsilon\right)$ it is clear that $x_{o}^{*} H\left(A_{1}\right) x_{o}=0, \ldots, x_{o}^{*} H\left(A_{n}\right) x_{o}=0$ and $x^{*} H\left(A_{1}\right) x=0, \ldots, x^{*} H\left(A_{n}\right) x=0$ for each $\quad x \in S\left(x_{o}, \varepsilon\right)$ and also $x_{o}^{*} S\left(A_{1}\right) x_{o}=0, \ldots, x_{o}^{*} S\left(A_{n}\right) x_{o}=0$ and $x^{*} S\left(A_{1}\right) x=0, \ldots, x^{*} S\left(A_{n}\right) x=0$ for each $x \in S\left(x_{o}, \varepsilon\right)$ thus by Theorem (3.1) we have $H\left(A_{1}\right), H\left(A_{2}\right), \ldots, H\left(A_{n}\right)$ and $S\left(A_{1}\right), S\left(A_{2}\right), \ldots, S\left(A_{n}\right)$ are both positive semi-definite and negative semidefinit and this implies that $H\left(A_{1}\right)=S\left(A_{1}\right)=0, \ldots, H\left(A_{n}\right)=S\left(A_{n}\right)=0$, then $A_{1}=H\left(A_{1}\right)+i S\left(A_{1}\right)=0, \ldots, A_{n}=H\left(A_{n}\right)+i S\left(A_{n}\right)=0$.

\section{Theorem 3.3}

Let $A_{1}, A_{2}, \ldots A_{n} \in M_{n}$ and $x_{o}$ be a unit vector such that $x_{0}{ }^{*} A_{1} x=x_{0}{ }^{*} A_{2} x=\ldots=x_{0}{ }^{*} A_{n} x=0$ belongs to the joint numerical range of $A_{1}, A_{2}, \ldots, A_{n}$, then zero is a sharp point of joint numerical range of $\mathrm{A}_{1}, \mathrm{~A}_{2}, \ldots, \mathrm{A}_{\mathrm{n}}$ if and only if there exists $\varepsilon>0, \quad \phi_{1}$ and $\phi_{2}$ such that $\phi_{1} \leq \arg \left(x^{*} A_{1} x\right) \leq \phi_{2}, \phi_{1} \leq \arg \left(x^{*} A_{2} x\right) \leq \phi_{2} \ldots$, $\phi_{1} \leq \arg \left(x^{*} A_{n} x\right) \leq \phi_{2}$ with $\phi_{2}-\phi_{1}<\pi$ for all $x \in S\left(x_{o}, \varepsilon\right)$.

Proof:

Suppose there exists $\varepsilon>0, \varphi_{1}$ and $\varphi_{2}$ such that $\varphi_{1} \leq \arg \left(x * A_{1} x\right) \leq \varphi_{2}$, $\varphi_{1} \leq \arg \left(x * A_{2} x\right) \leq \varphi_{2}, \ldots, \varphi_{1} \leq \arg \left(x * A_{n} x\right) \leq \varphi_{2}$. If $w_{1}=\frac{\pi}{2}-\varphi_{1}$ and $w_{2}=\frac{3 \pi}{2}-\varphi_{2}$ 
then $0<\mathrm{w}_{2}-\mathrm{w}_{1}<\pi$, and for the matrices $e^{i w} A_{1}+e^{-i w} A_{1}{ }^{*}, e^{i w} A_{2}+e^{-i w} A_{2}{ }^{*}$, $\ldots, e^{i w} A_{n}-e^{-i w} A_{n}{ }^{*}$

We have $\quad x_{0}{ }^{*}\left(e^{i w} A_{1}+e^{-i w} A^{*}{ }_{1}\right) x_{0}=0, \quad x_{0}{ }^{*}\left(e^{i w} A_{2}+e^{-i w} A^{*}{ }_{2}\right) x_{0}=0$,

$x_{0}{ }^{*}\left(e^{i w} A_{n}+e^{-i w} A^{*}{ }_{n}\right) x_{0}=0 \quad, \quad$ and $\operatorname{Re}\left(x^{*} e^{i w} A_{1} x\right)=\frac{1}{2} x^{*}\left(e^{i w} A_{1}+e^{-i w} A_{1}{ }^{*}\right) x \leq 0$,

$\operatorname{Re}\left(x^{*} e^{i w} A_{2} x\right)=\frac{1}{2} x^{*}\left(e^{i w} A_{2}+e^{-i w} A_{2}{ }^{*}\right) x \leq 0, \ldots$,

$\operatorname{Re}\left(x^{*} e^{i w} A_{n} x\right)=\frac{1}{2} x^{*}\left(e^{i w} A_{n}+e^{-i w} A_{n}^{*}\right) x \leq 0$,

for any $w \in\left[w_{1}, w_{2}\right]$ and for all $\mathrm{x} \in S\left(x_{0}, \varepsilon\right)$. Therefore by theorem (3.1) the matrices $e^{i w} A_{1}+e^{-i w} A_{1}{ }^{*}, e^{i w} A_{2}+e^{-i w} A_{2}{ }^{*}, \ldots, e^{i w} A_{n}+e^{-i w} A_{n}{ }^{*}$ are

non-negative semi definite and $\max \left\{x^{*}\left(e^{i w} A_{1}+e^{-i w} A_{1}{ }^{*}\right) x:\|x\|=1\right\}=0$, $\max \left\{x^{*}\left(e^{i w} A_{2}+e^{-i w} A_{2}{ }^{*}\right) x:\|x\|=1\right\}=0, \ldots, \max \left\{x^{*}\left(e^{i w} A_{n}+e^{-i w} A_{n}{ }^{*}\right) x:\|x\|=1\right\}=0$, for any $w \in\left[w_{1}, w_{2}\right]$ thus $\max \left\{\operatorname{Rez}: \mathrm{z} \in e^{i w} J N R\left(A_{1}, A_{2}, \ldots, A_{n}\right)\right\}=0$, this means that the origin is a sharp point of the joint numerical range of $A_{1}, A_{2}, \ldots, A_{n}$. Conversely, assume that zero is a sharp point of the joint numerical range of $A_{1}, A_{2}, \ldots, A_{n}$, then by the definition of sharp point there exists $w_{1}$ and $w_{2}$ belongs to $[0,2 \pi]$, such that for each $\mathrm{w}$ belongs to $\left[\mathrm{w}_{1}, \mathrm{w}_{2}\right]$,

$\max \left\{\operatorname{Rez}: \mathrm{Z} \in J N R\left(e^{i w}\left(A_{1}, A_{2}, \ldots, A_{n}\right)\right)\right\}=0$, where $w_{1}=\frac{\pi}{2}-\varphi_{1}$ and $w_{2}=\frac{3 \pi}{2}-\varphi_{2}$, so this implies that $\max \left\{x^{*}\left(e^{i w} A_{1}+e^{-i w} A_{1}^{*}\right) x:\|x\|=1\right\}=0, \ldots$,

$\max \left\{x^{*}\left(e^{i w} A_{n}+e^{-i w} A_{n}{ }^{*}\right) x:\|x\|=1\right\}=0$, because $x^{*}\left(e^{i w} A_{1}+e^{-i w} A_{1}{ }^{*}\right) x=$ $\operatorname{Re}\left(x^{*} e^{i w} A_{1} x\right), \ldots, x^{*}\left(e^{i w} A_{n}+e^{-i w} A_{n}{ }^{*}\right) x=\operatorname{Re}\left(x^{*} e^{i w} A_{n} x\right)$, we obtain that $\arg \left(x * A_{1} x\right), \arg \left(x * A_{2} x\right), \ldots, \arg \left(x^{*} A_{n} x\right)$ belongs to $\left[\varphi_{1}, \varphi_{2}\right]$ where $\varphi_{2}-\varphi_{1}=\left(\frac{3 \pi}{2}-w_{2}\right)-\left(\frac{\pi}{2}-w_{1}\right)<\pi$.

\section{Theorem 3.4}

Let $\lambda_{0}$ be a sharp point of the joint numerical range of the linear pencil $A_{1} \lambda_{1}-B_{1}, A_{2} \lambda_{2}-B_{2}, \ldots, A_{m} \lambda_{m}-B_{m}$. Then zero is a sharp point of $J N R\left(A_{1} \lambda_{o}-B_{1}, A_{2} \lambda_{o}-B_{2}, \ldots, A_{m} \lambda_{o}-B_{m}\right)$.

\section{Proof:}

By the equality $J N R\left(A_{1}\left(\lambda_{1}+\lambda_{o}\right)-B_{1}, A_{2}\left(\lambda_{2}+\lambda_{o}\right)-B_{2}, \ldots, A_{m}\left(\lambda_{m}+\lambda_{o}\right)-B_{m}\right)=$ $J N R\left(A_{1} \lambda_{1}-B_{1}, A_{2} \lambda_{2}-B_{2}, \ldots, A_{m} \lambda_{m}-B_{m}\right)-\lambda_{o}$, since $\lambda_{o}$ is a sharp point of $J N R\left(A_{1} \lambda_{1}-B_{1}, A_{2} \lambda_{2}-B_{2}, \ldots, A_{m} \lambda_{m}-B_{m}\right)$. This implies that zero is a sharp point 
of $J N R\left(A_{1} \lambda_{1}+\left(A_{1} \lambda_{o}-B_{1}\right), A_{2} \lambda_{2}+\left(A_{2} \lambda_{o}-B_{2}\right), \ldots, A_{m} \lambda_{m}+\left(A_{m} \lambda_{o}-B_{m}\right)\right)$, thus there exists a vector $x_{o}$ such that $x_{o}^{*}\left(A_{1} \lambda_{o} \quad B_{1}\right) x_{o}=0, \ldots, x_{o}^{*}\left(A_{m} \lambda_{o} \quad B_{m}\right) x_{o}=0$ and $x_{o}^{*} A_{1} x_{o}=k_{1} \neq 0, \ldots, x_{o}^{*} A_{m} x_{o}=k_{m} \neq 0$. It is not possible to have $x_{o}^{*}\left(A_{1} \lambda_{o}-B_{1}\right) x_{o}=x_{o}^{*} A_{1} x_{o}=0, x_{o}^{*}\left(A_{2} \lambda_{o}-B_{2}\right) x_{o}=x_{o}^{*} A_{2} x_{o}=0, \ldots, x_{o}^{*}\left(A_{m} \lambda_{o}-B_{m}\right) x_{o}=$ $x_{o}^{*} A_{m} x_{o}=0$ because $J N R\left(A_{1} \lambda_{1}+A_{1} \lambda_{o}-B_{1}, A_{2} \lambda_{2}+A_{2} \lambda_{o}-B_{2}, \ldots, A_{m} \lambda_{m}+A_{m} \lambda_{o}-B_{m}\right)$

$=C^{n}$. Since zero is a sharp point of $J N R\left(A_{1} \lambda_{1}+A_{1} \lambda_{o}-B_{1}, A_{2} \lambda_{2}+A_{2} \lambda_{o}-B_{2}\right.$, $\ldots, A_{m} \lambda_{m}+A_{m} \lambda_{o}-B_{m}$ ), there exists $r_{1}, r_{2}, \ldots, r_{m}>0$ such that for any complex number

$$
\begin{aligned}
& \gamma_{x_{1}}=\frac{-x^{*}\left(A_{1} \lambda_{o}-B_{1}\right) x}{x^{*} A_{1} x} \in S\left(0, r_{1}\right) \cap\left[\lambda_{1}<A_{1} x, x>+\lambda_{0}<A_{1} x, x>-B_{1}\right] \\
& \gamma_{x_{2}}=\frac{-x^{*}\left(A_{2} \lambda_{o}-B_{2}\right) x}{x^{*} A_{2} x} \in S\left(0, r_{2}\right) \cap\left[\lambda_{2}<A_{2} x, x>+\lambda_{0}<A_{2} x, x>-B_{2}\right] . \\
& \cdot \\
& \gamma_{x_{m}}=\frac{-x^{*}\left(A_{m} \lambda_{o}-B_{m}\right) x}{x^{*} A_{m} x} \in S\left(0, r_{m}\right) \cap\left[\lambda_{\mathrm{m}}<A_{m} x, x>+\lambda_{0}<A_{m} x, x>-B_{m}\right] .
\end{aligned}
$$

We have $\quad \phi_{1} \leq \arg \left(\frac{-x^{*}\left(A_{1} \lambda_{o}-B_{1}\right) x}{x^{*} A_{1} x}\right) \leq \phi_{2}$

$$
\phi_{1} \leq \arg \left(\frac{-x^{*}\left(A_{m} \lambda_{o}-B_{m}\right) x}{x^{*} A_{m} x}\right) \leq \phi_{2}
$$

with each $\phi_{2}-\phi_{1}<\pi$.Moreover, by the continuity of the functions $F_{11}(x)=x^{*} A_{1} x, F_{12}(x)=x^{*} A_{2} x, \ldots, F_{1 m}(x)=x^{*} A_{m} x$ and $F_{21}(x)=x^{*}\left(A_{1} \lambda_{o}-B_{1}\right) x$, ..., $F_{2 m}(x)=x^{*}\left(A_{m} \lambda_{o}-B_{m}\right) x$ for any $\varepsilon>0$ there exists a neighborhood $S\left(x_{o}, \delta\right)$ such that for any $x \in S\left(x_{o}, \delta\right) \quad x^{*} A_{1} x, x^{*} A_{2} x, \ldots, x^{*} A_{m} x$ belong to $S\left(k_{1}, \varepsilon\right), \ldots, S\left(k_{m}, \varepsilon\right)$ respectively and $\gamma_{x_{1}}, \gamma_{x_{2}}, \ldots, \gamma_{x_{m}}$ belong to $\mathrm{S}_{1}\left(0, \mathrm{r}_{1}\right), \mathrm{S}_{2}\left(0, \mathrm{r}_{2}\right), \ldots, \mathrm{S}_{\mathrm{m}}\left(0, \mathrm{r}_{\mathrm{m}}\right)$ respectively.

thus by equation $\arg (\mathrm{x} * \mathrm{~A} 1 \mathrm{x})+\arg \left(\gamma_{x_{1}}\right)=\arg \left(\mathrm{x}^{*}\left(\mathrm{~A}_{1} \lambda_{0}-\mathrm{B}_{1}\right) \mathrm{x}\right), \arg \left(\mathrm{x}^{*} \mathrm{~A}_{2} \mathrm{x}\right)+$ $\arg \left(\gamma_{x_{2}}\right)=\arg \left(\mathrm{x}^{*}\left(\mathrm{~A}_{2} \lambda_{0}-\mathrm{B}_{2}\right) \mathrm{x}\right), \ldots, \arg \left(\mathrm{x}^{*} \mathrm{~A}_{\mathrm{m}} \mathrm{x}\right)+\arg \left(\boldsymbol{\gamma}_{\mathbf{x}_{\mathrm{m}}}\right)=\arg \left(\mathrm{x}^{*}\left(\mathrm{~A}_{\mathrm{m}} \boldsymbol{\lambda}_{\mathbf{0}}-\mathrm{B}_{\mathrm{m}}\right) \mathrm{x}\right)$, we have that each of $\arg \left(x^{*}\left(A_{1} \lambda_{o}-B_{1}\right) x\right), \ldots, \arg \left(x^{*}\left(A_{m} \lambda_{o}-B_{m}\right) x\right)$ belongs to the $\left[\theta_{1}, \theta_{2}\right]$ for any $x \in S\left(x_{o}, \delta\right)$ and for suitable $\theta_{1}, \theta_{2}$ with $\theta_{2}-\theta_{1}<\pi$. And 
then by Theorem (3.3) it is clear that zero is a sharp point of the $J N R\left(A_{1} \lambda_{o}-B_{1}, A_{2} \lambda_{o}-B_{2}, \ldots, A_{m} \lambda_{o}-B_{m}\right)$.

Degenerate cases of sharp points are the isolated points, and we have the following statement:

\section{Theorem 3.5}

Let $p(\lambda)=A_{m} \lambda^{m}+A_{m-1} \lambda^{m-1}+\ldots+A_{1} \lambda+A_{0}$ be an nxn matrix polynomial such that zero does not belong to the joint numerical range $J N R\left(A_{m}\right)$ of the leading coefficient matrix. If $\lambda_{o}$ is an isolated point of the joint numerical range $\operatorname{JNR}\left(p\left(\lambda_{o}\right)\right)$ then $p\left(\lambda_{o}\right)=0$.

\section{Proof:}

Assume $\lambda_{o}=0$ then there exists a unit vector such that $<A_{j} x, x>=0, j=0,1,2, \ldots, m$. This means that $\left\langle A_{0} x, x>=0\right.$, now if $\left.<A_{0} y, y\right\rangle=0$ for any unit vector $\mathrm{y}$ then the matrix $A_{o}$ satisfies $A_{o}=0$. We assume that there exists a unit vector $\mathrm{y}$ with $\left\langle A_{j} y, y\right\rangle \neq 0 ; j=0,1,2, \ldots, m$ i.e. $<A_{o} y, y>\neq 0$. We consider a polynomial $Q(\lambda, t)=<A_{m}\left(\cos \frac{t \pi}{2} x+\sin \frac{t \pi}{2} y\right),\left(\cos \frac{t \pi}{2} x+\sin \frac{t \pi}{2} y\right)>\lambda^{m}+\ldots+<A_{1}\left(\cos \frac{t \pi}{2} x+\sin \frac{t \pi}{2} y\right)$, $\left(\cos \frac{t \pi}{2} x+\sin \frac{t \pi}{2} y\right)>\lambda+<A_{o}\left(\cos \frac{t \pi}{2} x+\sin \frac{t \pi}{2} y\right),\left(\cos \frac{t \pi}{2} x+\sin \frac{t \pi}{2} y\right)>$. This satisfies $<A_{m}\left(\cos \frac{t \pi}{2} x+\sin \frac{t \pi}{2} y\right),\left(\cos \frac{t \pi}{2} x+\sin \frac{t \pi}{2} y\right)>\neq 0$, because zero does not belong to the joint numerical range of the leading coefficient $A_{m}$. Now it is sufficient to prove that there exists a sequence $\left(t_{n}\right)$ for which $t_{n}$ tends to zero as $\mathrm{n}$ tends to infinity and $Q\left(\lambda_{n}, t_{n}\right)=0$, at first we fix $0<\mathrm{t}<1$ the condition $Q(0, t)=<A_{o}\left(\cos \frac{t \pi}{2} x+\sin \frac{t \pi}{2} y\right),\left(\cos \frac{t \pi}{2} x+\sin \frac{t \pi}{2} y\right)>=0$, is equal to $<A_{o}\left(x+\tan \left(\frac{t \pi}{2}\right) y, x+\tan \left(\frac{t \pi}{2}\right) y>=0\right.$ on the other hand we consider the function $\left.<A_{o}(x+s y), x+s y\right\rangle=s$, where we have $\left\langle A_{o} y, y\right\rangle \neq 0$ by the choice of the unit vector $\mathrm{y} .\left\langle A_{o} x, y\right\rangle+\left\langle A_{o} y, x\right\rangle \neq 0$ then we have $\left\langle A_{o}(x+s y), x+s y\right\rangle \neq 0$

for sufficiently small $\mathrm{s}>0$ and if $\left\langle A_{o} x, y\right\rangle+\left\langle A_{o} y, x\right\rangle \neq 0$ then we have $\left\langle A_{o}(x+s y), x+s y\right\rangle=s^{2}\left\langle A_{o} y, x\right\rangle \neq 0$. Thus we conclude that $Q(0, t) \neq 0$ for a sufficiently small $\mathrm{t}>0$ so that $\left\langle A_{o}\left(x+\tan \left(\frac{t \pi}{2}\right) y, x+\tan \left(\frac{t \pi}{2}\right) y\right\rangle \neq 0\right.$. Hence the equation $\mathrm{Q}(\lambda, \mathrm{t})=0$ in $\lambda$ has $\mathrm{m}$ roots by the fundamental theorem of algebra. The roots of the algebraic equation depends continuously on the coefficient, hence $\mathrm{P}(0)=\mathrm{A}_{0}=0$ 


\section{REFERENCES}

[1] Au-Yeung, Y. H. and Tsing, N.K "An extention of the Hausdorff Toeplitz theorem on the numerical range" Proc.Amer. Math. Soc., 89(1983),pp.215-218

[2] Binding, P, Farenick, D. and Li, C.K."A dilation and norm in several variable operators theory" Canada. J. Math.,47(1995), pp. 449-461.

[3] Binding, P. and Li, C. K. "Joint numerical range of Hermitian matrices and simultaneous diagonalization", Linear Algebra Appl., 151(1991), pp.157-168.

[4] Cho, M and Takaguchi, M. "Some classes of commuting m-tuples of operators", Studin Math. 80(1984) pp. 245-259.

[5] Fan, M. and Tits, A. "m-form numerical range and the computation of the structured singular" IEEE Trans. Automat Control AC, 33(1988), pp.184-289.

[6] Horn, R.A and Johnson, C.R, "Topics in Matrix Analysis" Cambridge University Press, 1991.

[7] Li, C.K. and Rodman, L. "Numerical range of matrix polynomials" SIAM J. Matrix Anal. Appl. 15 (1994), pp.1256-1265.

[8] Maroulas, J. and Psarrakos, P. "Geometrical properties of numerical range of matrix polynomials" comput, Math. Appl. 31(1996), pp.41-47.

[9] Psarrakos, P. J. and Tsatsomeros, M.J. "On the relation between the numerical range and the joint numerical range and of matrix polynomials", the Electronic Journal of Linear Algebra, 6 (2000), pp.20-30. 